\title{
Platinum speciation and transport in sulfur-rich hydrothermal fluids
}

\author{
C. LASKAR ${ }^{1 *}$, G.S. POKROVSKI ${ }^{1}$, M. KOKH ${ }^{1}$, J.L. \\ HAZEMANN $^{2}$, E.F. BAZARKINA ${ }^{2}$, E. DESMAELE ${ }^{3}$ \\ AND R. VUILLEUMIER ${ }^{3}$
}

${ }^{1}$ Géosciences Environnement Toulouse (GET), UPS, CNRS, IRD, OMP, 31400, Toulouse, France

(*correspondence : clement.laskar@get.omp.eu)

${ }^{2}$ Institut Néel, UGA, CNRS, 38042, Grenoble, France

${ }^{3}$ Ecole Normale Supérieure (ENS), UPMC, CNRS, Sorbonne Universités, 75005, Paris, France

Understanding PGE deposit formation requires knowledge of the chemical state and mobility of platinum group elements (PGE) in magmatic-hydrothermal fluids. Yet, existing data on aqueous chloride, sulfate, and hydroxide complexes of PGE predict far too small metal contents $(<\mathrm{ppt}$ to $\mathrm{ppb}$ ) in fluids from most geological settings, thus calling upon an important role of sulfide complexes [1-3], and, potentially, trisulfur ion ligands in PGE transport [4]. In an attempt to quantify the effect of sulfur on PGE mobility, here we combined solubility measurements, in-situ X-ray absorption spectroscopy (XAS), thermodynamic (TD) and molecular dynamics (MD) modeling, based on recent advances of our knowledge of sulfur speciation in crustal fluids [5]. Our new data at $300^{\circ} \mathrm{C}$ and 500 bar suggest formation of two main complexes transporting Pt in the fluid at concentrations of $10 \mathrm{~s}$ ppm over a wide $\mathrm{pH}$ range (4-8): $\mathrm{Pt}(\mathrm{HS})_{4}{ }^{2-}$ in hydrogen sulfide $\mathrm{H}_{2} \mathrm{~S} / \mathrm{HS}^{-}$solutions, and $\mathrm{Pt}(\mathrm{HS})_{2}\left(\mathrm{~S}_{3}\right)_{2}{ }^{2-}$ in sulfide-sulfate $\mathrm{H}_{2} \mathrm{~S}_{/} / \mathrm{SO}_{4}{ }^{2-} / \mathrm{S}_{3}{ }^{--}$solutions. Notably, the obtained concentrations of $\mathrm{Pt}(\mathrm{HS}) 4^{2-}$ in $\mathrm{H}_{2} \mathrm{~S} / \mathrm{HS}^{-}$ solutions, using an improved protocol for accurate fluid sampling from a flexible-cell reactor, are 1000 times higher than those reported in previous quench-based studies [6]. TD and MD simulations, based on combined solubility and insitu XAS data confirm the large stability of the $\mathrm{Pt}(\mathrm{HS})_{2}\left(\mathrm{~S}_{3}\right)_{2}{ }^{2-}$ complex, analogous to that proposed for gold [4], with Pt(II) as the major oxidation state. Thus, our new data highlight an important role of previously ignored sulfur complexes that significantly enhance PGE transport by ore-forming fluids in the Earth's crust.

[1] Bazarkina et al. (2014) GCA 146, 107-131. [2] Kokh et al. (2017) GCA 197, 433-466. [3] Tagirov et al. (2019) GCA 254, 86-101. [4] Pokrovski et al. (2015) PNAS 112, 13484 13489. [5] Pokrovski and Dubessy (2015) EPSL 411, 298309. [6] Gammons and Bloom (1993) GCA 57, 2451-2467. 